\title{
ASSESSMENT OF THE PERIPHERAL BLOOD FLOW OF LOWER LIMBS IN PATIENTS WITH DIABETES MELLITUS WITH THE USE OF DIGITAL PHOTOPLETHYSMOGRAPHY
}

\author{
Hubena G., Osina O., Prindesova Busikova J., Vasicko T. \\ Department of Occupational Medicine and Toxicology, Comenius University, Jessenius Faculty of Medicine and \\ Martin University Hospital, Martin, Slovakia
}

\begin{abstract}
A b s t r a c t
Introduction: The microcirculation of the lower limbs (LL) of diabetics is influenced by hyperglycemia and several factors (hypertension, obesity, dyslipidemia) leading to the functional and later the structural changes, manifesting as dysregulation of the peripheral blood flow.

Materials and Methods: The study included 39 patients with type 2 diabetes, and the same number of the control group. The digital photoplethysmography (PPG) was used for assessment of the peripheral circulation with reflectance mode, sensing from the 1st and 2nd toe of the LL after acclimatisation of the patient in the supine position. The subjects were also asked to refrain from smoking, to avoid drinks containing caffeine and using of medicaments with vasodilatator function at least 2 hours before examination.

Results: The pulse amplitudes and the peak times of the PPG curves were significantly higher in diabetic group than in the control group. The mean pulse amplitudes in diabetics were in the range of $0.69 \% 0.86 \%$, the pulse peak time in the range of $140-154 \mathrm{~ms}$. The mean pulse amplitudes in the control group were in the range of 0.37 $0.54 \%$, the mean pulse peak time were in the range of $120-133$ ms. The PPG findings of the LL were symmetrical (non-significant t-test). We have not found a correlation between the PPG records and duration, compensation of diabetes, age, glycemia, blood pressure, dyslipidemia, smoking and obesity.

Conclusion: This simple-to-use technique shows the increased total skin microcirculation in diabetic patients. Microvascular shunting of blood presenting in peripheral nerves and in the skin of diabetic feet are responsible for reduced hyperemia response to any inflammatory process with the increased susceptibility for inquiring the diabetic foot infection.
\end{abstract}

Key words: diabetic microangiopathy, digital photoplethysmography, pulse amplitude, peak time

\section{INTRODUCTION}

Diabetes mellitus is a chronic vascular disease leading to a variety of serious complications. One of the most threatening complications of diabetes is the diabetic foot syndrome, responsible for $60 \%$ of all non-traumatic amputations of the lower limbs (1). It is still the most common cause of hospitalization of the diabetic patients in developed countries (2).

Long-term hyperglycemia leads to a damage of the vascular tissue through the development of the endothelial dysfunction which is an important part in the pathogenesis of the diabetic microangiopathy (3). This process is connected with the decreased biological availability of nitric oxide with subsequent limited vasodilatator function (4). The most pronounced microvascular disturbances appear in the lower limbs, which is also the site of the most severe complications (1) probably due to increased hydrostatic pressure of this locality (5).

Other risk factors of development of the endothelial dysfunction are genetic predisposition, obesity, smoking, hypertension, and dyslipidemia. The final effect is initially a complex of functional and later structural changes that will cause the dysregulation of the blood flow, cell growth, thereby leading to a proliferation of connective tissue overgrowth and extravascular materials as well as thickening of basement membranes (2).

Address for correspondence:

MUDr. Hubena Gabriela, Kollarova Str. 81, 036 01Martin, Slovakia E-mail: gabriela.hubena@gmail.com; Phone: 0915866475 
The impairment of a blood flow through the vasa nervorum (microangiopathy) leads to the development of the diabetic neuropathy. With the loss of sympathetic tonus there occurs a dilatation of arterio-venous (AV) shunts with a redistribution of blood flow from nutrient capillaries into subpapillary vessels with lower resistance with subsequent development of the chronic capillary ischemia $(1,3)$. So the peripheral microvasculature will be incapable of increasing nutritive capillary flow to the required degree in response to, for example, injurious stimulus (6). Clinically there is an image of warm, well-perfused limbs usually with the swelling in the dorsum (1). Impaired tissue perfusion due to abnormality of the microvasular system is common among other risk factors, such hypertension, diabetes, obesity and dyslipidemia, associated in metabolic syndrome.

The atherosclerotic process in diabetes mellitus is morphologically not significantly different from atherosclerotic changes in non-diabetics, but has the special characteristics: frequent and earlier occurrence with more rapid progression, diffuse, multi-segmental vascular involvement with collaterals in contrast to intersegmental, unilateral disease in the non-diabetic patients $(6,7)$. According to a several authors there haven $t$ been found a statistically significant differences between the basal microvascular flow of the lower limbs of patients with diabetes in comparison with non-diabetics with varying degrees of peripheral arterial disease (8). Our aim was to determine changes in peripheral microcirculation of the lower limbs in diabetics in comparison to the healthy population.

\section{MATERIALS AND METHODS}

\section{Patients}

Our sample consisted of 39 diabetic patients, 33 men and 6 women, with a mean age of 53 years (range of $44-58$ years) with a confirmed diagnosis of type 2 diabetes mellitus with no signs of diabetic ulcer. Healthy subjects of the control group, 33 men and 6 women with a mean age 50 years (range of 45 - 55 years), were free from cardiovascular, neurological diseases, without anamnesis of the ischemia or injury of the lower limbs. They had a systolic blood pressure less than $140 \mathrm{mmHg}$ and diastolic blood pressure less than $90 \mathrm{mmHg}$ and glycemia under $5.9 \mathrm{mmol} / 1$ (upper limit of laboratory in Martin University Hospital). The healthy subjects were without permanent medication, cardiovascular, neurological diseases and ischemia and injury of lower limbs. In first contact with patient we have collected detailed information on each subject s medical history, laboratory findings, smoking habit and intake of medications. Characteristics of the diabetic and control group are shown in the Table 1. The glycemia in diabetic group was in the range of $6.6-13.9 \mathrm{mmol} / \mathrm{l}$, glycated hemoglobin in the range of $4-11.1 \mathrm{mmol} / 1$ with duration of diabetes from 0 - 17 years (divided to three subgroups - 0 years, $0.25-5$ years, 6 - 17 years). Glycated hemoglobin has not been examined in control in case of its indicational restriction in healthy subjects. Glycated hemoglobin was available in 31 patients (divided in two subgroups $4-6.5 \%, 6.57$ - $11.1 \%$, LDL and HDL - lipoproteins were available in 37 patients, creatinine in 30 diabetic patients. 
Table 1 Characteristics of the diabetic group and the control group

\begin{tabular}{|c|c|c|}
\hline Parameter & $\begin{array}{l}\text { Diabetic group } \\
\quad(\mathrm{n}=39)\end{array}$ & $\begin{array}{l}\text { Control group } \\
\quad(\mathrm{n}=39)\end{array}$ \\
\hline Gender Male/Female & $39 / 6$ & $39 / 6$ \\
\hline Height $(\mathrm{cm})$ & $173.64 \pm 7.25$ & $174.9 \pm 7.59$ \\
\hline Weight (kg) & $98.49 \pm 15.39 *$ & $80.44 \pm 11.6$ \\
\hline BMI $\left(\mathrm{kg} / \mathrm{m}^{2}\right)$ & $32.6 \pm 4.25^{*}$ & $26.21 \pm 2.86$ \\
\hline Smokers / non - smokers & $11 / 28$ & $9 / 30$ \\
\hline Age (years) & $53.23 \pm 3.67$ & $50.38 \pm 3.1$ \\
\hline Systolic pressure (mm Hg) & $134.23 \pm 10.97^{*}$ & $125.77 \pm 9.57$ \\
\hline Diastolic pressure (mm Hg) & $84.74 \pm 6.17$ & $82.05 \pm 5.7$ \\
\hline Glucose (mmol/l) & $8.15 \pm 2.41^{*}$ & $5.24 \pm 0.33$ \\
\hline Duration of diabetes (years) & $5.21 \pm 4.89$ & - \\
\hline $\mathrm{HbA}_{1} \mathrm{c}(\%) / \mathrm{n}=31+$ & $6.69 \pm 1.75$ & - \\
\hline Total cholesterol (mmol/l) & $5.49 \pm 1.38$ & $5.86 \pm 0.91$ \\
\hline Triglycerides (mmol/l) & $2.89 \pm 2.33^{*}$ & $1.57 \pm 0.63$ \\
\hline $\mathrm{HDL}(\mathrm{mmo} / \mathrm{l}) \mathrm{n}=37+$ & $1.20 \pm 0.28^{*}$ & $1.53 \pm 0.38$ \\
\hline $\mathrm{LDL}(\mathrm{mmol} / \mathrm{l}) \mathrm{n}=31+$ & $3.03 \pm 0.83$ & $3.37 \pm 0.82$ \\
\hline Creatinine $n=30+$ & $97.63 \pm 14.24$ & - \\
\hline
\end{tabular}

Legend: BP - blood pressure; BMI - body mass index; $\mathrm{HbA}_{1} \mathrm{c}$ - glycated hemoglobin; HDL - high density lipoproteins, LDL - low density lipoproteins*; statistical significance at $\mathrm{p}<0.05,+$ number of examined subjects with laboratory parameter

\section{Photoplethysmography (PPG)}

Photoplethysmography is an optical, non-invasive method for studies of the blood volume pulsations. The finger sensor consists of photodiode and detector, emitting and receiving an infrared light that penetrates the tissue under the probe and it is reflected by red blood cells. The appearance of the photoplethysmographic waveform is commonly divided into two phases: the anacrotic phase is the rising edge of the pulse during the systole, whereas the catacrotic phase is falling edge of the pulse in diastole (9). In the proximal part of the catacrotic part is the dicrotic notch which is a sign of healthy compliant arteries. The pulse amplitude is an indicator of the pulsatile changes in blood volume caused by arterial blood flow around the measurement site (9). Another characteristic of the PPG is the peak time, which is a time from the beginning of the anacrotic part to the peak of the waveform. In practice it is usually used the visual analysis of the photoplethysmogram s contour. The values of the pulse amplitudes and peak times of the lower limbs of a healthy persons haven $t$ been standardized yet. 


\section{Measurement protocol}

The photoplethysmographic modul CDS 1000 with the reflectance mode-photoplethysmography was used to examination. The examination was realized at standard conditions after automatic calibration of the signal through the patient $\mathrm{s}$ tissue before every measuring ( 1 minute calibration). The room temperature was kept constant at around $22-24^{\circ} \mathrm{C}$. The measuring was obtained from the 1 st and the 2nd toe of both legs. Examination was automatic with the graphic record in computer. Measurement in every subject lasted approximately $5 \mathrm{~min}$. The subjects were in the supine position, relaxed and acclimatized for approximately 15 minutes before the examination. Examined subjects were not allowed to smoke, to drink beverages with the caffeine and to use the medicaments with vasoactive effects at least two hours before the examination. Every patient had the blood pressure measured, the hands were placed a side the body, motionless.

\section{Statistical analysis}

The results are expressed as mean \pm standard deviation (SD). The mean values of pulse amplitudes and pulse peak times, assessed by means of PPG in diabetic patients and control group, were compared using a parametric two sample t-test in MS Excel 2010. A probability of $\mathrm{p}<0.05$ was assumed as significant. Pearson's correlation test was used to obtain correlations between the pulse amplitudes, peak times and age, BMI, blood pressure, duration of diabetes and laboratory findings of both groups.

\section{RESULTS}

We have found significantly higher values of amplitudes and the pulse peak times in diabetic group in comparison with the control group at statistically significant level p $<0.05$. The mean pulse amplitudes in diabetics were in the range of $0.69 \% \quad 0.86 \%$, the pulse peak times in the range of $140-154 \mathrm{~ms}$. the mean pulse amplitudes in the control group were in the range of $0.370 .54 \%$, the mean pulse peak time were in the range of $120-133 \mathrm{~ms}$ (Fig. 1 and Fig. 2). The photoplethysmographic records of lower limbs have been symmetrical for the 1st and 2nd toe of both lower limbs (non-significant t-test).

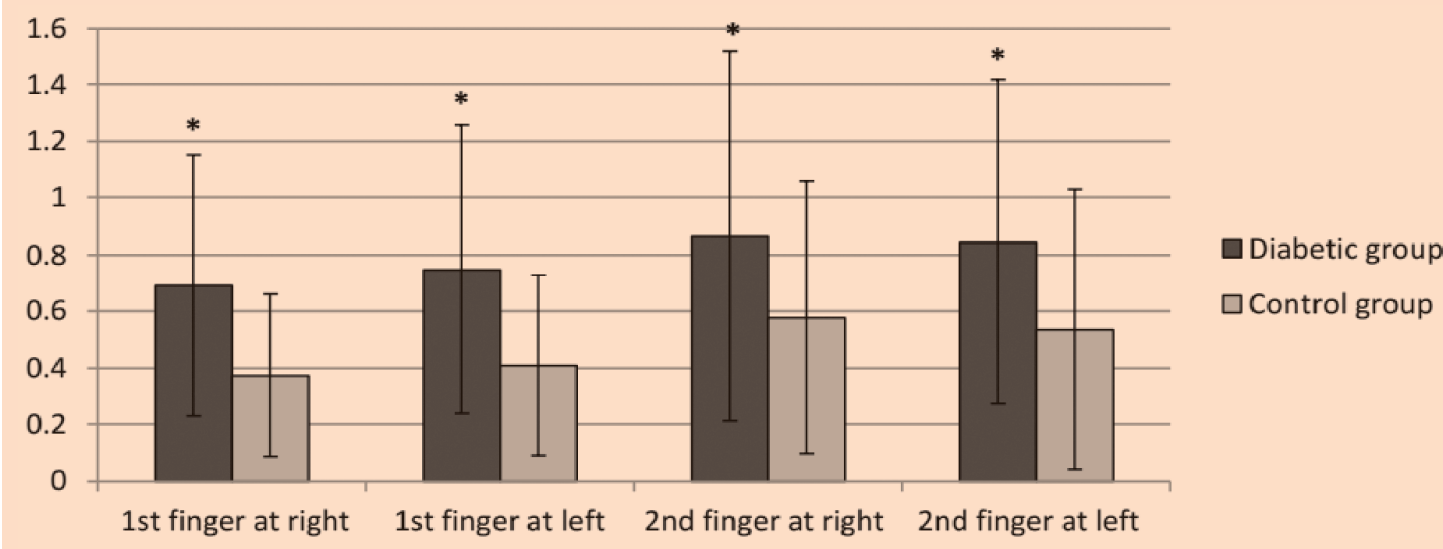

Fig. 1 The mean \pm SD values of the photoplethysmographic amplitudes of the lower limbs (1st and 2nd toe) in the diabetic and in the control group; * statistical significance at $\mathrm{p}<0.05$ 


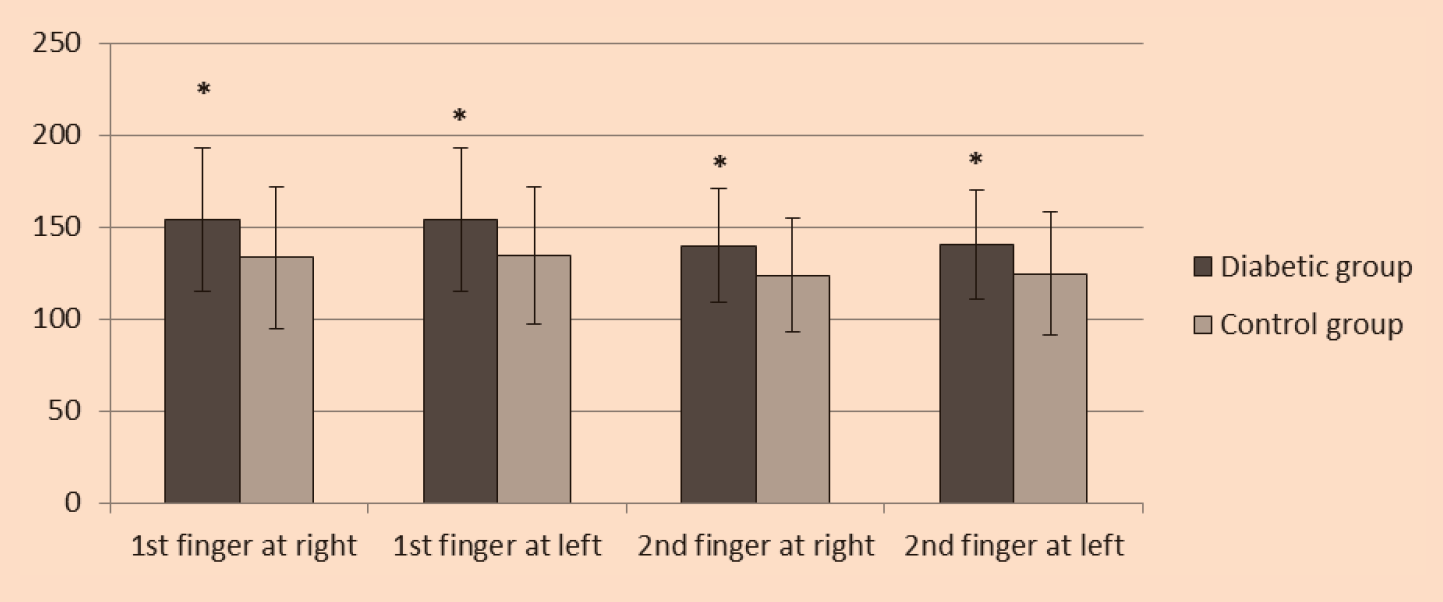

Fig. 2 The mean values \pm SD of the photoplethysmographic peak times of the lower limbs (1st and 2nd toe) in the diabetic and in the control group; * statistical significance at $\mathrm{p}<0.05$

Patients with type 2 diabetes mellitus in our study had significant signs of metabolic syndrome (Tab. 1). $71.8 \%$ of the diabetics were obese, $79.5 \%$ had an arterial hypertension, $90 \%$ dyslipidemia. $20.5 \%$ had a chronic ischemic heart disease, 5.1\% of them had a stroke and $7.7 \%$ a myocardial infarction in the medical history. $71.8 \%$ of the diabetics were on antihypertensive therapy, $53.9 \%$ on the lipid-lowering drugs and $46.2 \%$ of them on the antiagreggant therapy. A family history of diabetes was positive in $51.3 \%$ of patients and for cardiovascular disease in $71.8 \%$ of patients. The treatment of diabetes is shown in the Tab. 2 .

Tab. 2 Treatment of diabetic patients

\begin{tabular}{|l|c|c|}
\hline Treatment & N & $\%$ \\
\hline Oral antidiabetic drugs (OADs) & 22 & $56 \%$ \\
\hline Diet & 6 & $15 \%$ \\
\hline Oral antidiabetic drugs (OADs) + insulin & 4 & $10 \%$ \\
\hline Insulin & 3 & $8 \%$ \\
\hline Newly diagnosed DM & 3 & $8 \%$ \\
\hline Oral antidiabetic drugs (OADs) + incretins & 1 & $3 \%$ \\
\hline
\end{tabular}

The correlations of the amplitudes and peak times of PPG curves with glycemia, glycated hemoglobin, BMI, triglycerides, cholesterol, LDL and HDL - lipoproteins and the blood pressure have not been confirmed in our study (data not shown). We have not found statistically significant differences of the pulse amplitudes and peak times in patients with diabetes in terms of duration and compensation of the diabetic disease.

We also have not found a correlation of the blood pressure (BP) in the diabetic group with the amplitudes and the peak times (non-significant t-test between the subgroup of diabetic 
patients with systolic BP 110 - $130 \mathrm{mmHg}$ and 135 - $160 \mathrm{~mm} \mathrm{Hg}$, and the subgroup of control $110-120 \mathrm{~mm} \mathrm{Hg}$ and $125-130 \mathrm{~mm} \mathrm{Hg}$, diastolic BP was almost uniform within the groups - it have not been compared). We have found significant differences between the pulse amplitudes and peak times of PPG curves at the comparable systolic and diastolic blood pressure between the groups (data not shown). Significant differences in values of amplitudes and peak times within the lower and higher creatinaemia have not been found. The mean value of creatinine in diabetics was in the normal range.

Diabetes group consisted of 28 non-smokers (72\%) and 11 smokers (28\%), a control group of 30 non-smokers (77\%) and 9 smokers (29\%). We have not found statistically significant differences between the pulse amplitudes and peak times of smokers and non-smokers (Tab. 3). The values of the pulse amplitudes of smokers in the diabetic group have been higher than in the control group of non-smokers, which copied the results of the PPG records of the whole diabetic group in comparison with the control group. The values of peak times of the PPG curves within and between smokers and non-smokers have been analogical (data not shown).

Tab. 3 The mean \pm amplitudes of PPG curves of the 1st and 2nd toe of the lower limbs in smokers and non-smokers of both groups

\begin{tabular}{|l|c|c|c|c|c|c|}
\hline Toe/side & \multicolumn{2}{|c|}{ Diabetic group } & $\begin{array}{c}\text { P values } \\
\text { (A vs B) }\end{array}$ & \multicolumn{2}{c|}{ Control group } & $\begin{array}{c}\text { P values } \\
\text { (C vs D) I }\end{array}$ \\
\hline & $\begin{array}{c}\text { Smokers } \\
\text { N }=11\end{array}$ & $\begin{array}{c}\text { Nonsmokers } \\
\text { N }=28\end{array}$ & & $\begin{array}{c}\text { Smokers } \\
\text { N }=9\end{array}$ & $\begin{array}{c}\text { Nonsmokers } \\
\text { N = 30 }\end{array}$ & \\
\hline & A & B & & C & D & \\
\hline $1^{\text {st }}$ toe, right & 0.655 & $0.691^{*}$ & NS & 0.407 & 0.372 & NS \\
\hline $2^{\text {nd }}$ toe, right & 0.795 & $0.864^{*}$ & NS & 0.605 & 0.537 & NS \\
\hline $1^{\text {st }}$ toe, left & 0.888 & $0.749^{*}$ & NS & 0.418 & 0.386 & NS \\
\hline $2^{\text {nd }}$ toe, left & 0.776 & $0.844^{*}$ & NS & 0.516 & 0.497 & NS \\
\hline
\end{tabular}

* $\mathrm{p}<0.05$ compared with group D (controls); NS - not significant

\section{DISCUSSION}

Our results have shown that peripheral blood flow of the lower limbs detected through the digital PPG was significantly higher in the diabetic group than in the control group. Numerous studies have suggested that in the presence of peripheral neuropathy arteriovenous shunt flow is increased which has led to the assumption that a microvascular "steal“ of blood away from the capillary bed may be occurring (6). Loss of sympathetic tonus causes a dilation of arterio-venous (AV) shunts with a redistribution of blood flow from nutrient capillaries into subpapillary vessels with lower resistance and subsequent development of chronic capillary ischemia.

The PPG method is unable to distinguish between the flows in the AV shunts vessels and the nutritional capillaries in the skin (10), so there is a good circulation despite the chronic capillary ischemia.

The presence of diabetic neuropathy we should expect in every patient with type 1 diabetes mellitus with duration of the diabetes more than five years and in every patient with 
type 2 diabetes mellitus regardless of the length of disease, because the type 2 diabetes is usually diagnosed with a significant delay in comparison with the actual time of development the disease (10). Some neuropathic changes have been detected already in the stage of impaired glucose tolerance (11). So we have not realized neurological assessment of diabetic patients what is the limitation of this study.

The diabetic group had significant higher values of the systolic and diastolic blood pressure than the control group. In our study we have not found significant differences between the pulse amplitudes and peak times of PPG curves at comparable values of systolic and diastolic blood pressure within the groups, but we have found significant differences between the pulse amplitudes and peak times of PPG curves at the comparable systolic and diastolic blood pressure between the groups (data not shown). It indicates that not only the blood pressure is responsible for increased microvascular flow. In normotensive patients with 2 type diabetes other mechanisms contribute to the markedly reduced vasodilatory reserve (6).

Structural alterations in small resistance vessels is more pronounced in patients with both diabetes mellitus and hypertension, thus suggesting that clustering of risk factors may have synergic deterious effects on vasculature (13). The correlation between the blood pressure and photoplethysmographic parameters has not been found in our study. The values of amplitudes and peak times have not been changed according to the values of creatinine. The high values of amplitudes and peak times in diabetics have not been caused by hypertension due to a nephropathy.

We have not found correlation between a duration and compensation of diabetes with the photoplethysmographic parameters. Diabetes, even in its early phase, is characterised by a hyperdynamic circulation linked to insulin resistance and several investigations have found abnormally elevated blood flow in early period of diabetes (14). In this study there was a tendency to have higher amplitudes of PPG curves in the beginning of diabetes mellitus than in longer duration of diabetes but this difference has not been statistically significant. The values of amplitudes and peak times of PPG according to glycated haemoglobin have not been statistically significant.

Generally, it is known that the photoplethysmographic waveform is age-dependent. With the older age the amplitudes are decreasing and peak times are increasing due to higher stiffness of arteries which was confirmed in the PPG studies of Spigulis et al., Menzlova, Jayasree et al. etc. $(15,16,17)$.

Patients of both groups were approximately in the same age, also the blood flow has been higher in the diabetic group. This findings were presented in our study but we haven t found a statistical significant differences of the parameters of PPG waveforms in relation to age. Advanced age is associated with impaired arterial elasticity and endothelial function and in addition, hyperglycemia and many other risk factors, such high blood pressure $190.6 \%$ of diabetics in study), dyslipidemia ( $90 \%$ of diabetics in study), obesity ( $72 \%$ of diabetics in study), genetic predisposition accelerating the process of atherosclerotic changes. In our study, the majority of diabetic patients were obese with significant signs of metabolic syndrome $(71.8 \%$ of diabetics in this study) (Tab. 1). It is noteworthy that microvascular changes that result from one risk factor could predispose to other risk factors (18). Functional microangiopathy could be an integral part of the metabolic syndrome (14). Tissue metabolism and local microflow are usually tightly coupled, in such a way that flow adapts to the metabolic demand of the surrounding tissue so it has been increasingly suggested that maladapted arteriolar reactivity may impair nutrient delivery and lead to aggravate insulin resistance (14).

We have not found a correlation between the amplitudes and peak times of the PPG curves and glycemia or glycated hemoglobin, BMI, cholesterol, triglycerides, HDL and LDL-lipoproteins. Our patients with diabetes were in therapy with good compensation of diabetic disease, they have on antihypertensive, hypolipidemic and antiagregant treatment. The question was, whether the increased values of amplitudes, peak time weren t related to using 
vasodilatators with long lasting effect, such calcium blocators (used by $28 \%$ of diabetics). We have not found statistically significant differences in amplitudes a peak times of PPG curves between patients with treatment with calcium blocators and patients without this treatment.

Smoking is also responsible for the progression of microvascular and microvascular complications. The statistically significant differences between pulse amplitudes and peak times of smokers and non-smokers have not been found in our study. Pulse amplitudes and peak times of waveforms of non-smokers from diabetic group have been significantly higher than in a control group of smokers (Tab. 3).

Endothelial dysfunction is thought to play an important role not only in the initiation of atherosclerosis, but also in its progression and clinical sequelae. It can be assumed that diabetics in our group had varying degrees of atherosclerosis with increased value of the peak time of the PPG curves. Diabetics in our study were without intermittent claudication, limitation of this study is missing of macroangiopathy exclusion. According to several studies, such the study of Urbancic-Rovan et al. that non-significant differences in foot microvascular flow between the subgroups with low and normal ABI supports the hypothesis that PAOD does not have a significant impact on skin microcirculation in the basal state, neither in diabetic patients nor in non-diabetic subjects (10).

It was confirmed that improved glycemic control can delay the progression of diabetic microangiopathy, but not of macroangiopathy, due to mentioned factors above together with smoking, aging, physical inactivity and family history of premature cardiovascular events, which are involved in the pathogenesis of accelerated atherosclerosis. This could indicate that macro- and miro-vascular disease of the lower extremities do not proceed simultaneously (8).

PPG is used mainly in qualitative assessment through the shape of waveforms because there have not been determined the universal standard conditions for evaluation of the photoplethysmographic parameters, especially of lower limbs yet.

The aim of the study was quantitative assessment of photoplethysmographic records with finding the correlation between PPG and different factors which have not been confirmed in our study. Limitation of this study is the possibility of parallel presence of diabetic neuropathy and macroangiopathy, which have not been excluded by neurological examinations and by measurement of ankle-brachial index.

This method provides assessment of a total peripheral blood flow, nowadays it has been superseded by other methods (laser Doppler flowmetry). This method provides other diagnostic possibilities mainly in addition to other modes of PPG to better vascular examination (modul of laser Doppler, modul for ankle-brachial pressure index (ABPI), venous occlusion modul etc.).

\section{CONCLUSIONS}

It can be concluded that the amplitudes of the PPG curves of diabetic patients ranging between $0.7 \%$ and $0.9 \%$, the peak times between $140-154 \mathrm{~ms}$, findings were symmetrical. Our results have shown increased peripheral blood flow of the lower limbs in diabetic patients with significantly higher values of amplitudes and peak times of PPG curves than in the control group. Microvascular shunting of blood presenting in peripheral nerves and in the skin of diabetic feet are responsible for reduced hyperaemia response to any inflammatory process with the increased susceptibility for inquiring the diabetic foot infection. It is essential that the patients at risk for the development of diabetic microvascular complications are identified earlier. 


\section{REFERENCES}

1. Jorneskog G, Brismar K, Fagrell B. Pronounced skin capillary ischemia in the feet of diabetic patient s with bad metabolic control. Diabetologia. 1998; 41(4): 410 - 415

2. Svacina S et al. Cevni komplikace diabetu. Edice Symposium 10. Praha: Galen; 2005, 150 p.

3. Popescu RM, Cotutiu C, Graur M, Caruntu I. Vascular and nerve lesions of the diabetic foot - a morphological study. In Romanian Journal of Morphology and Embyryology. 2010; 51(3):483-488

4. Galajda P. Metabolicky syndrom, kardiovaskularne a metabolicke rizika. In Via practica. 2007, vol. 4 (S4): 5 - 9

5. Gavornik P. Diabeticka angiopatia - etiopatogeneza a klinicke prejavy (hypersyndrom X). In Bratisl Lek Listy. 2000; 101(10): $569-576$

6. Tooke J. Peripheral microvascular disease in diabetes. In Diabetes Res Clin Pract 1996; 30, Suppl. 1, p. 61 - 65

7. Mokan M, Martinka E, Galajda P, et al. Diabetes mellitus a vybrané metabolicke ochorenia. Martin: Vydavatelstvo P+M, 2008. 1003 p.

8. Urbancic - Rovan V. et al. Macro and microcirculation in the lower extremities - Possible relationship. In Diabetes Res Clin Pract. 2006; 73 (2): 166 - 173

9. Elgendi M. On the analysis of fingertip photoplethysmogram signal. In Curr Cardiol Rev. $2012 ; 8$ (1): $14-25$

10. Chittenden S, Shami SK. Microvascular investigations in diabetes mellitus. In Postgrad Med J. $1993 ; 69$ (812): $419-428$

11. Akbari CM, Logerfo FW. Diabetes and peripheral vascular disease. In J Vasc Surg. 1999; 30 (2): 373 - 384

12. Grofik M et al. Neurologicke prejavy diabetes mellitus. In Neurol prax. 2005; 6 (1): 28 - 33

13. Rizzoni D, Rosei EA. Small artery remodelling in diabetes mellitus. In Nutr Metabol Cardiovasc Dis. $2009 ; 19$ (8): $587-592$

14. Wiernsperger NF, Bouskela E. Microcirculation in insulin resistance and diabetes: more than just a complication. In Diabetes Metab. 2003; 29: 6S77 - 6S87

15. Spigulis J, Kukulis I, Fridenberga E, Venckus G. Potential of advanced photoplethysmography sensing for noninvasive vascular diagnostics and early screening. Proc. SPIE 4625, Clinical Diagnostic Systems: Technologies and Instrumentation, 2012; 38 (June 7, 2002)

16. Menzlova M. Diagnosticke moznosti prstove pletyzmografie. In Dermatol.prax. 2008; 4:188-192

17. Jayasree VK, Sandhya TV, Radhakrishnan P. Non-invasive studies on age related parameters using a blood volume pulse sensor. In Measurement Science Review, 2008; 8 (4): 82 -86

18. Levy BI, et al. Impaired tissue perfusion: A pathology common to hypertension, obesity, and diabetes mellitus. In Circulation. 2008, 118 (9): 968 - 976

Received: March, 25, 2015

Accepted: July, 7, 2015 